\title{
Quantitative contrast-enhanced ultrasound for monitoring vedolizumab therapy in inflammatory bowel disease patients: a pilot study
}

Acta Radiologica

2018, Vol. 59(I0) II49-II56

(C) The Foundation Acta Radiologica 2018

Article reuse guidelines:

sagepub.com/journals-permissions DOI: I0.1 |77/0284|85 | I7752032 journals.sagepub.com/home/acr @SAGE

\author{
Ruediger S Goertz, Daniel Klett, Dane Wildner, Raja Atreya, \\ Markus F Neurath and Deike Strobel
}

\begin{abstract}
Background: Microvascularization of the bowel wall can be visualized and quantified non-invasively by software-assisted analysis of derived time-intensity curves.

Purpose: To perform software-based quantification of bowel wall perfusion using quantitative contrast-enhanced ultrasound (CEUS) according to clinical response in patients with inflammatory bowel disease treated with vedolizumab.

Material and Methods: In a prospective study, in 18 out of 34 patients, high-frequency ultrasound of bowel wall thickness using color Doppler flow combined with CEUS was performed at baseline and after 14 weeks of treatment with vedolizumab. Clinical activity scores at week 14 were used to differentiate between responders and non-responders. CEUS parameters were calculated by software analysis of the video loops.

Results: Nine of 18 patients (I I with Crohn's disease and seven with ulcerative colitis) showed response to treatment with vedolizumab. Overall, the responder group showed a significant decrease in the semi-quantitative color Doppler vascularization score. Amplitude-derived CEUS parameters of mural microvascularization such as peak enhancement or wash-in rate decreased in responders, in contrast with non-responders. Time-derived parameters remained stable or increased during treatment in all patients.

Conclusion: Analysis of bowel microvascularization by CEUS shows statistically significant changes in the wash-in-rate related to response of vedolizumab therapy.
\end{abstract}

\section{Keywords}

Crohn's disease, ulcerative colitis, vedolizumab, quantification, contrast-enhanced ultrasound, CEUS

Date received: 8 September 2017; accepted: II December 2017

\section{Introduction}

Crohn's disease (CD) and ulcerative colitis (UC) are the main entities of inflammatory bowel disease (IBD) and yield significant morbidity. Recently, Vedolizumab (Entyvio ${ }^{\circledR}$; Takeda Pharmaceutical, Osaka, Japan), a monoclonal antibody selective for $\alpha 4 \beta 7$ integrin, showed therapeutic efficacy in a subgroup of refractory patients, with likely higher efficacy in UC than in CD (1). To monitor disease activity and to determine response to anti-inflammatory therapy, several diagnostic options are available. Besides clinical disease activity assessment, evaluation by endoscopy is the reference that is quite elaborate due to invasiveness, sedation, pain, and prior necessity for colonic purge. In addition to magnetic resonance imaging (MRI) or computed tomography (CT), B-mode ultrasound measuring bowel wall thickness and using color Doppler flow

Department of Internal Medicine I, Friedrich-Alexander-University Erlangen-Nuernberg, Erlangen, Germany

\section{Corresponding author:}

Ruediger S Goertz, Department of Internal Medicine I, FriedrichAlexander-University Erlangen-Nuernberg, Ulmenweg 18, 91054

Erlangen, Germany.

Email: ruediger.goertz@uk-erlangen.de 
has shown great potential and is used as the primary diagnostic method in daily routine (2).

$\mathrm{CD}$ and UC show increased perfusion and blood volume of the bowel wall in cases with high disease activity $(3,4)$. With the aid of dynamic contrast enhancedultrasound (CEUS), microvascularization of the bowel wall can be visualized and quantified non-invasively by software-assisted analysis of derived time-intensity curves (5). Analysis of CEUS can be longitudinally compared between different examinations without discomfort (6-8). The intravenously applied ultrasound contrast agent is eliminated via the lungs and undesired side-effects (radiation, kidney failure, and thyroid problems) of contrast-enhanced CT do not exist (9).

In our study, parameters of bowel wall perfusion were evaluated using CEUS to monitor clinical therapeutic outcomes in patients with IBD treated with vedolizumab.

\section{Material and Methods}

In this prospective, observational pilot study between August 2014 and May 2015, a total of 34 patients with proven IBD that presented for initiation of vedolizumab therapy (therapy weeks $0,2,6$, and 14) were screened. This observational study was approved by the Ethics Committee at our institute and written informed consent for CEUS and for the study was obtained from all study patients. Sixteen patients out of the screened 34 patients were excluded from the study due to various reasons such as refusal, interruption of treatment before week 14 or CEUS video slopes of poor technical quality insufficient for software quantification (Fig. 1). In total, 18 patients (11 with CD and seven with UC) completed the study. At baseline (first application of vedolizumab) and after 14 weeks, B-mode ultrasound and CEUS examination were performed.

At the time of each vedolizumab treatment, blood samples (leukocytes, C-reactive-protein [CRP], and hemoglobin) and clinical scoring (Harvey-Bradshaw index $[\mathrm{HBI}]$ or clinical Mayo Subscore) were performed. Clinical data and patient characteristics (age, body mass index [BMI], former therapy, and duration of disease) were documented.

\section{Clinical scores}

The HBI clinically determined disease activity by five clinical criteria in patients with CD (10). A sum of $<5$ points indicated clinical remission, $5-7$ points mild disease, $8-16$ points moderate, and $>16$ points severe activity. In patients with UC, the clinical Mayo Subscore was determined (11) by three clinical items awarding a maximum of nine points. By means of changes in the clinical score, patients were classified

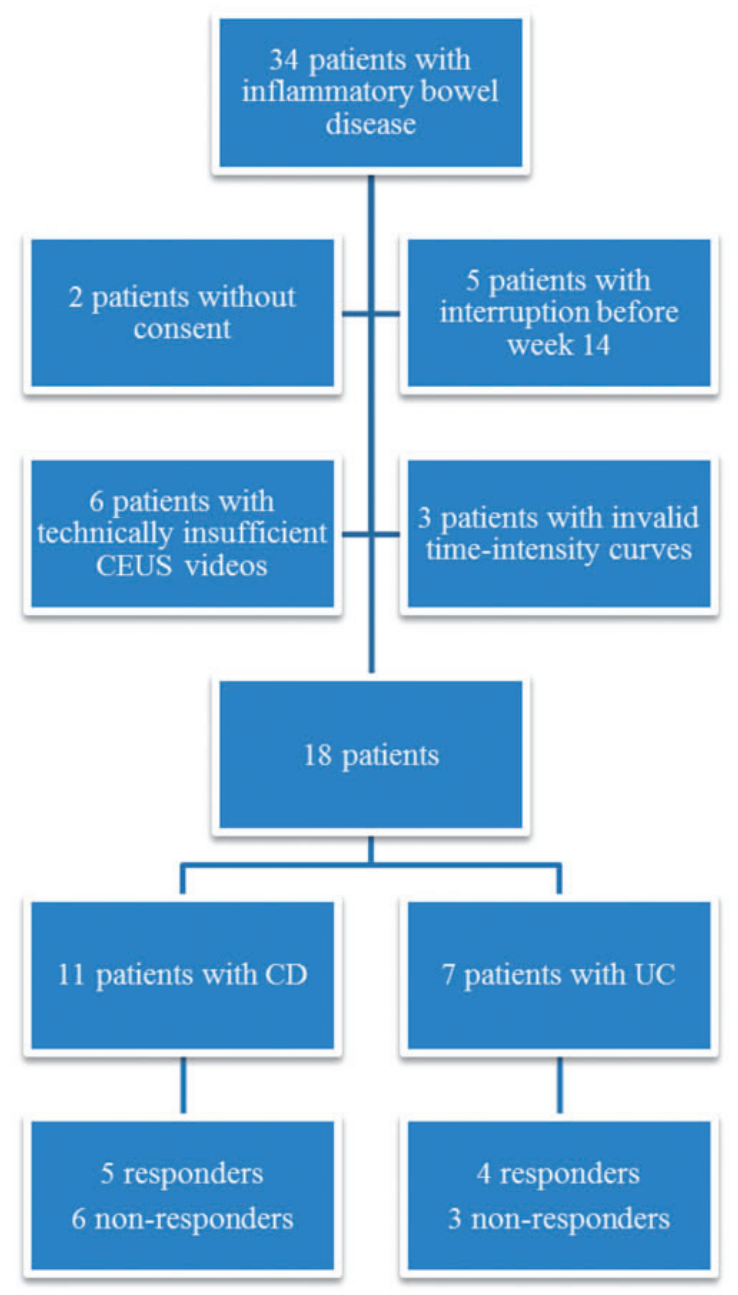

Fig. I. Flow chart of screened patients with IBD and final study cohort with Crohn's disease (CD) or ulcerative colitis (UC).

as group 1 "clinical response" (UC: Mayo Subscore $\Delta \leq-1$; CD: HBI $\Delta \leq-4$ or remission at week 14 , respectively, and continuation of vedolizumab therapy) or group 2 "no response" (other than group 1).

\section{Ultrasound and quantification}

B-mode high-frequency ultrasound with the linear transducer 9L4 and CEUS with the curved array transducer 6C1HD were performed using the Acuson S2000 (Siemens Medical Solution, software version VB21A, Erlangen, Germany). Three unblinded senior physicians with more than ten years of experience performed high-frequency ultrasound and CEUS in the most affected bowel segment. The same location was evaluated in the follow-up. Image size, gain, focus zone, and pulse-repetition frequency were optimized. The affected bowel segment was described regarding bowel wall thickness $(\mathrm{mm})$ and intramural semi-quantitative vascularization grade 0-3 (no color Doppler signal $=0$; 
few, short signals $=1$, long intramural perfusion signals $=2$, and intramural and mesentery signals $=3$ ) in a modified version reported by Limberg et al. (4) (Fig. 2).

CEUS was performed with the same settings before and during therapy: low mechanical index (0.04); lowest penetration depth; scale $\mathrm{D}$; frequency of 1.5 $\mathrm{MHz}$; time-gain compensation in middle position; and a dual window. An intravenous injection of a bolus of 2.4-mL ultrasound contrast agent (SonoVue ${ }^{\circledR}$, Bracco S.P.A., Milan, Italy) was administered followed by a $10-\mathrm{mL}$ flush of saline solution. The linear raw data of the examinations were recorded continuously over a period of $2 \mathrm{~min}$ with a frame rate of $16 \mathrm{~Hz}$ and exported as DICOM format to an external program called VueBox ${ }^{\circledR}$ (Bracco Suisse SA, Version 4.2.3.0, Software Applications, Geneva, Switzerland). A region of interest (ROI) was defined manually to the bowel wall transmurally in a longitudinal section (Fig. 3). Gas reflections or further artifacts were avoided by the shape of the ROI or by editing the clip.

The time-intensity curves generated by VueBox ${ }^{\circledR}$ were automatically analyzed to calculate CEUS parameters of bolus kinetics. These included amplitudederived parameters measured in arbitrary units (a.u.) as well as the time-derived parameters measured in seconds (Table 1). The wash-in rate/wash-out rate (WiR/WoR) is the maximum/minimum slope of the time-intensity curve represented as a tangent at the ascending/descending part of the curve.

\section{Statistical analysis}

All data were expressed as the mean \pm standard deviation and the range indicated in brackets. Spearman's correlation coefficient (r) was used for analysis of

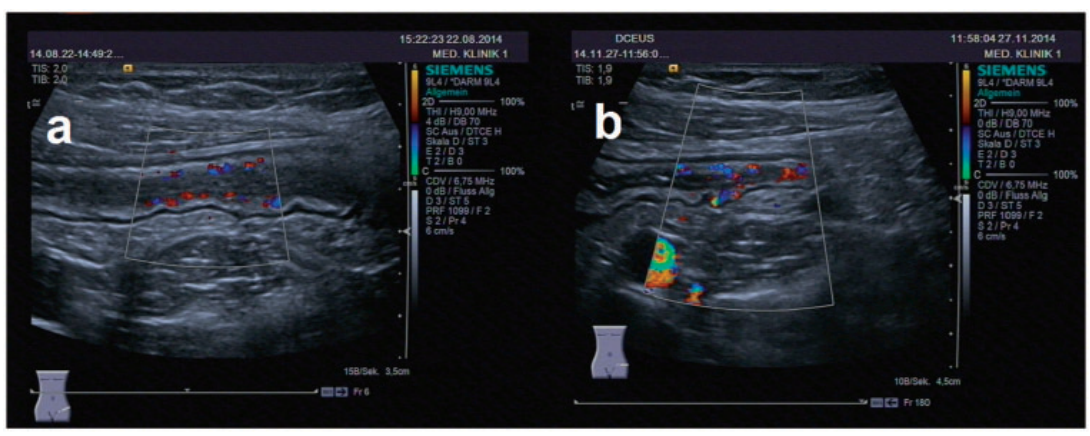

Fig. 2. (a, b) Patient with $C D$ with improvement of clinical ( $\mathrm{HBI}$ decreased from 18 to 6 points) and endoscopic activity during treatment: b-mode ultrasound with decrease of bowel wall thickness from 4.4 to $3.5 \mathrm{~mm}$, but persistend color doppler flow.

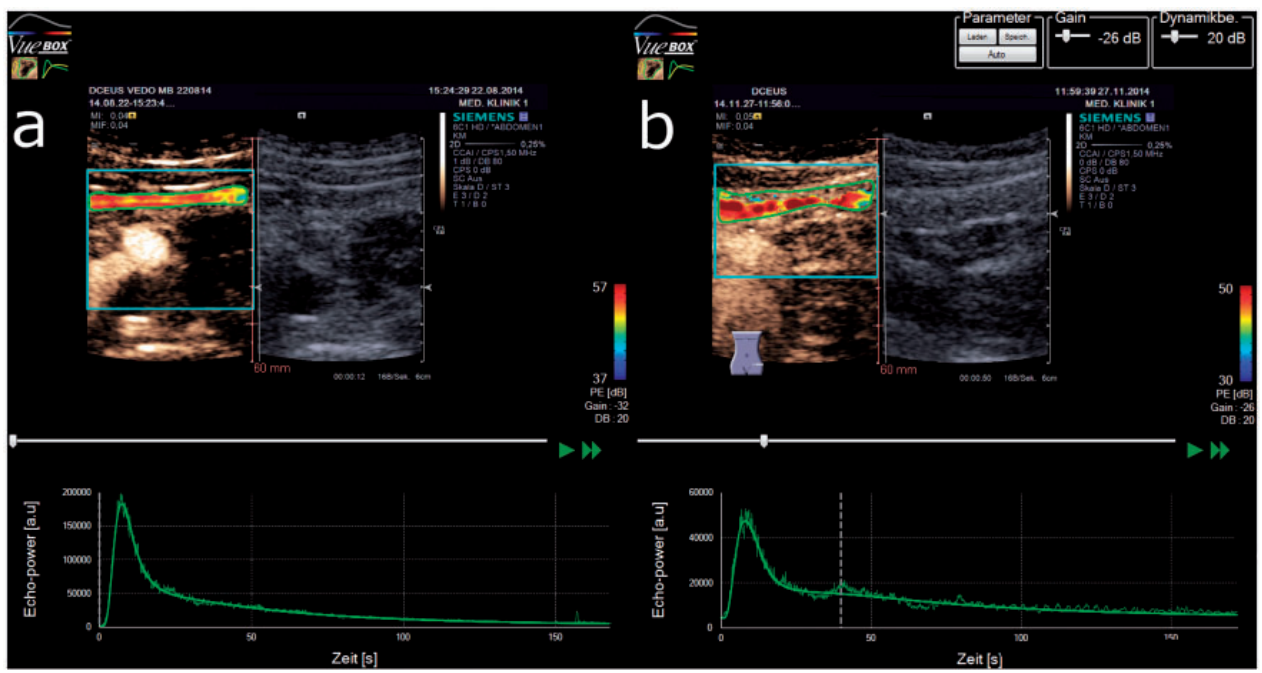

Fig. 3. (a, b) Same patient with CD seen in Fig. 2: CEUS at baseline and I 4 weeks later showing a decrease in peak enhancement (PE) from 181.257 to 42.975 a.u. 
Table I. Overview of amplitude- and time-derived parameters of CEUS quantification.

\begin{tabular}{ll}
\hline $\begin{array}{l}\text { Amplitude-derived parameters (a.u.) } \\
\text { PE }\end{array}$ & Peak enhancement \\
WiWoAUC & Wash-in and wash-out AUC \\
WiAUC & Wash-in AUC \\
WoAUC & Wash-out AUC \\
WiR & Wash-in rate \\
WoR & Wash-out rate \\
Time-derived parameters (s) & \\
TTP & Time to peak \\
RT & Rise time \\
FT & Fall time \\
mTTI & Mean transit time local \\
\hline
\end{tabular}

relationships between variables. For the comparison of related samples, the paired $t$ test was performed. In each patient, the percent change from each CEUS parameter measured at baseline and week 14 was calculated and presented related to therapeutic response. Results were illustrated in tables or in a typical boxplot. Small asterisks mark outliers. A $P$ value $<0.05$ indicated a statistically significant correlation or difference. All reported $P$ values were two-sided. Statistical and graphical analyses were performed using the Statistical Package for the Social Sciences (version 19.0.0.1, IBM SPSS statistics, Armonk, NY, USA).

\section{Results}

\section{Ulcerative colitis (UC)}

All seven patients ( 6 men, 1 woman; mean age $=51.0 \pm 9$ years) had sigmoiditis (Table 2). According to the clinical Mayo Subscore, two patients had high inflammatory activity, three had moderate activity, and two had mild disease activity at baseline. Four patients showed a favorable course of disease and formed group 1 including one patient with complete remission. At baseline, patients with UC showed a mean bowel wall thickness of $5.3 \pm 0.8 \mathrm{~mm}$ (range $=4.1-6.4$ ) and were higher than normal values. Under therapy, bowel wall thickness remained virtually constant with $5.1 \pm 1.3 \mathrm{~mm}$ at week 14. An increase (of $>1 \mathrm{~mm}$ ) was found in two patients. An increase of semi-quantitative vascularization of the bowel wall was found in only two patients with no therapeutic response (Table 3).

\section{Crohn's disease (CD)}

Eleven patients with CD ( 3 men, 8 women) had a mean age of $38.5 \pm 13$ years. All patients received biologic
Table 2. Patient characteristics of seven patients with ulcerative colitis (UC) and II patients with Crohn's disease (CD).

\begin{tabular}{lll}
\hline Patient & UC & CD \\
\hline Sex (female/male) & $\mathrm{I} / 6$ & $8 / 3$ \\
Mean age (years) & $5 \mathrm{I} \pm 9$ & $38.5 \pm \mathrm{I} 3$ \\
$\mathrm{BMI}\left(\mathrm{kg} / \mathrm{m}^{2}\right)$ & $24.9 \pm 4.6$ & $22.7 \pm 3.2$ \\
Disease duration (years) & $7.3 \pm 6$ & $\mathrm{I} 3 \pm 10$ \\
Prior treatment with & $7 \mathrm{I} .4$ & $\mathrm{I} 00$ \\
$\quad$ biologicals (\%) & & \\
Clinical score & Mayo Subscore & $\mathrm{HBI}$ \\
Baseline & $5.1 \pm \mathrm{I} .7$ & $\mathrm{II} .6 \pm 6.5$ \\
Week I4 & $4.0 \pm 2.5$ & $9.5 \pm 6.4$ \\
\hline
\end{tabular}

BMI, body mass index; HBI, Harvey-Bradshaw index.

Table 3. Evolution of bowel wall thickness and semi-quantitative color Doppler vascularization in Crohn's disease (CD) und ulcerative colitis (UC), mean and standard deviation.

\begin{tabular}{|c|c|c|c|c|}
\hline \multirow[b]{2}{*}{ Parameter } & \multicolumn{2}{|c|}{$\begin{array}{l}\text { Responders } \\
\text { group I }(\mathrm{n}=9)\end{array}$} & \multicolumn{2}{|c|}{$\begin{array}{l}\text { Non-responders } \\
\text { group2 }(\mathrm{n}=9)\end{array}$} \\
\hline & Baseline & Week I4 & Baseline & Week 14 \\
\hline \multicolumn{5}{|c|}{ Bowel wall thickness (mm) } \\
\hline All & $5.5 \pm 0.7$ & $5.1 \pm 1.6$ & $6.0 \pm 1.1$ & $5.9 \pm 1 . I$ \\
\hline$C D$ & $5.3 \pm 0.8$ & $5.3 \pm 1.8$ & $6.6 \pm 0.6$ & $6.1 \pm 1.2$ \\
\hline UC & $5.8 \pm 0.7$ & $4.8 \pm 1.6$ & $4.7 \pm 0.6$ & $5.6 \pm 0.7$ \\
\hline \multicolumn{5}{|c|}{ Vascularization (grade 0-3) } \\
\hline All & $1.9 \pm 0.9$ & $0.9 \pm 0.8^{*}$ & $\mathrm{I} .8 \pm 0.7$ & $1.9 \pm 0.9$ \\
\hline CD & $2.4 \pm 0.9$ & $\mathrm{I} .2 \pm 0.8$ & $2.0 \pm 0.6$ & $\mathrm{I} .5 \pm 0.8$ \\
\hline UC & $\mathrm{I} .3 \pm 0.5$ & $0.5 \pm 0.6$ & $\mathrm{I} .3 \pm 0.6$ & $2.7 \pm 0.6^{*}$ \\
\hline
\end{tabular}

$* \mathrm{P}<0.05$.

agents in the pre-study history, with $9 / 11(82 \%)$ receiving two different medical drugs. Four patients had a prior history of surgery (ileocecal resection). At baseline, two patients had severe disease activity, six had moderate activity, and one patient had mild activity. Two patients appeared to be in remission. At week 14 , five patients improved with a mean of $-8.4 \mathrm{HBI}$ from 14.6 to $6.2 \mathrm{HBI}$ points (two patients of them achieving remission status). One patient remained stable with high activity, and the remaining five patients deteriorated (corresponding to non-responders in group 2). The semi-quantitative color Doppler score was pathologic in all IBD patients at baseline with four patients showing highly elevated hypervascularization (Table 3). At week 14, only one patient remained at grade 3 . The decline of vascularization in the responder group failed to be significant $(P=0.056)$. 


\section{CEUS results of all patients}

At baseline, none of the CEUS parameters correlated with the clinical scores (HBI) in CD, but in UC the clinical Mayo Subscore correlated positively with WiWoAUC, WiAUC, WoAUC, and mTTI. In all 18 patients, bowel wall thickness did not correlate with CEUS parameters, whereas the semi-quantitative vascularization score correlated with some amplitudederived values such as PE $(\mathrm{r}=0.52, \quad P=0.029)$,
WiR, WoR, and WiPI, but not with time-derived CEUS parameters.

An analysis of the intra-individual percent changes of the kinetic parameters of all patients with $\mathrm{CD}$ or UC related to therapy response is shown in Table 4. In the responder group, the amplitude-derived parameters such as PE and WiAUC, for example, showed a trend to decrease in all patients and in patients with $\mathrm{CD}$ or UC, respectively (Fig. 4). Only the WiR showed

Table 4. Percent change (\%) in CEUS parameters between baseline evaluation and after I4 weeks of therapy: responders (group I) and non-responders (group 2) with Crohn's disease (CD) und ulcerative colitis (UC).

\begin{tabular}{|c|c|c|c|c|c|c|}
\hline \multirow{2}{*}{$\begin{array}{l}\text { Percent change (\%) } \\
\text { Parameter }\end{array}$} & \multicolumn{3}{|c|}{ Responders group I $(n=9)$} & \multicolumn{3}{|c|}{ Non-responders group $2(n=9)$} \\
\hline & All & $C D$ & UC & All & $C D$ & UC \\
\hline \multicolumn{7}{|c|}{ Amplitude-derived (a.u.) } \\
\hline PE & $-40.8 \pm 36$ & $-45.3 \pm 47$ & $-35.3 \pm 21$ & $61.4 \pm 189$ & $94.0 \pm 228$ & $-2.5 \pm 61.2$ \\
\hline WiWoAUC & $-29.0 \pm 45$ & $-46.0 \pm 52$ & $-7.8 \pm 26$ & $32.0 \pm 98$ & $47.9 \pm 115$ & $0.3 \pm 51$ \\
\hline WiAUC & $-30.2 \pm 47$ & $-43.1 \pm 60$ & $-14.1 \pm 22$ & $38.1 \pm 134$ & $58.2 \pm 164$ & $-1.9 \pm 40$ \\
\hline WoAUC & $-28.3 \pm 45$ & $-47.5 \pm 48$ & $-4.4 \pm 32$ & $31.3 \pm 88$ & $46.3 \pm 102$ & $1.2 \pm 57$ \\
\hline WiR & $-45.8 \pm 33^{*}$ & $-45.3 \pm 4 I^{*}$ & $-46.4 \pm 24^{*}$ & $125 \pm 314$ & $187.8 \pm 374$ & $-0.1 \pm 95$ \\
\hline WoR & $-42.7 \pm 46$ & $-40.4 \pm 54$ & $-45.6 \pm 4 I$ & $170.5 \pm 402$ & $251.4 \pm 482$ & $8.8 \pm 90$ \\
\hline Wipi & $-40.9 \pm 35$ & $-45.7 \pm 46$ & $-35.0 \pm 19$ & $58.6 \pm 18 \mid$ & $90.2 \pm 217$ & $-4.8 \pm 62$ \\
\hline \multicolumn{7}{|l|}{ Time-derived (s) } \\
\hline TTP & $12.0 \pm 39$ & $-0.8 \pm 4 I$ & $28.0 \pm 33$ & $24.5 \pm 52$ & $16.9 \pm 54$ & $39.6 \pm 54$ \\
\hline mTTI & $14.7 \pm 23$ & $16.7 \pm 30$ & $12.2 \pm 16$ & $-10.3 \pm 32$ & $-0.2 \pm 32$ & $-30.4 \pm 26$ \\
\hline RT & $|5| \pm 4 \mid$. & $-3.4 \pm 26$ & $38.3 \pm 49$ & $18.7 \pm 57$ & $20.8 \pm 66$ & $14.6 \pm 43$ \\
\hline FT & $28.4 \pm 66$ & $-0.9 \pm 23$ & $65.0 \pm 88$ & $31.5 \pm 88$ & $39.2 \pm 100$ & $16.0 \pm 74$ \\
\hline
\end{tabular}

$* \mathrm{P}<0.05$

(a)

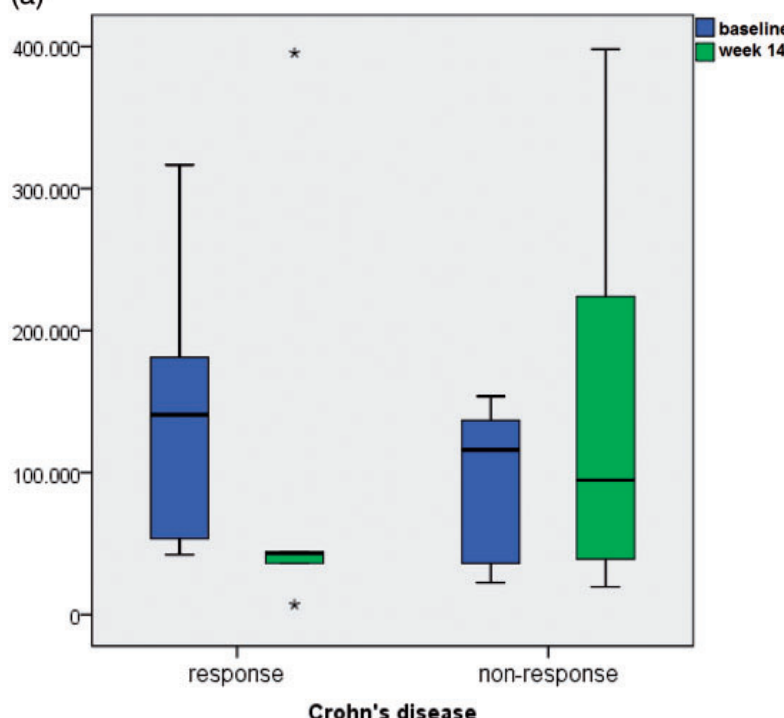

(b)

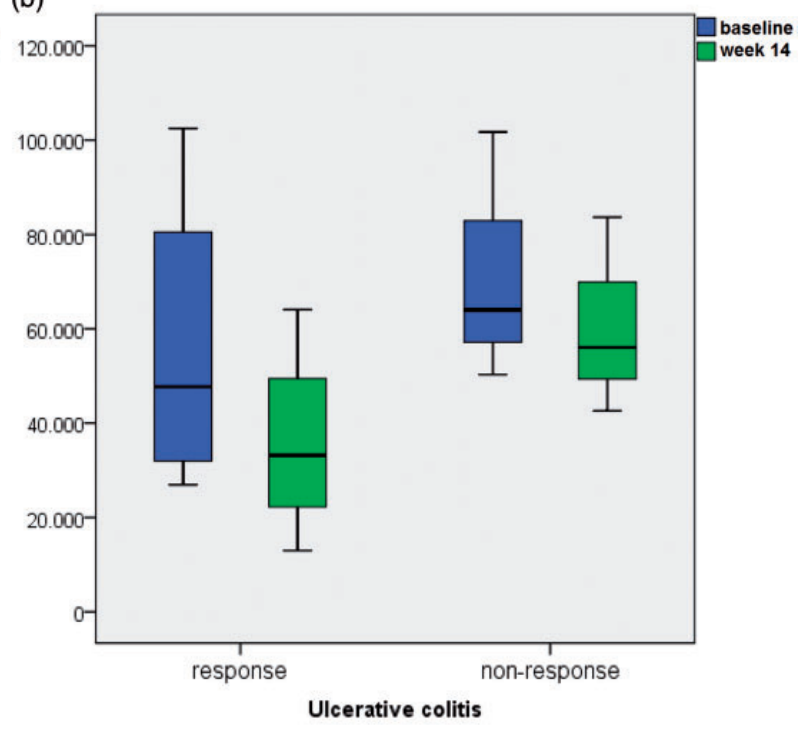

Fig. 4. Decrease of CEUS PE in responders and minor changes in non-responders at baseline and week I4 in patients with (a) CD and (b) UC. 
a statistically significant decrease in all responders $(P=0.016)$ and in group 1 patients with $\mathrm{CD}$ and $\mathrm{UC}$ ( $P=0.038$ and $P=0.037$, respectively). In the nonresponder group, the amplitude-derived parameters increased overall and in the $\mathrm{CD}$ group. In patients with $\mathrm{UC}$, these parameters tended to remain stable. The time-derived parameters such as TTP, RT, and FT increased, but with no consistent tendency related to response.

\section{Discussion}

Our study evaluating CEUS quantification of the bowel wall in patients with CD and UC at baseline and after 14 weeks of treatment with vedolizumab showed a significant change in semi-quantitative color Doppler flow in high-frequency ultrasound and CEUS kinetic parameters. In particular, amplitude-derived CEUS parameters showed a relevant decrease in the response group in patients with $\mathrm{CD}$ and $\mathrm{UC}$. The semi-quantitative color Doppler score showed a positive association with amplitude-derived parameters.

B-mode parameters such as bowel wall thickness and color Doppler intensity classification have revealed differences in active and inactive disease that correlated with endoscopic evaluation, but not or weakly with clinical activity scores in CD (12-15). Histopathological inflammation or high vascular density has been found significantly correlated with active disease on endoscopy, baseline ultrasonography, and CEUS with a higher PE and a shorter TTP $(16,17)$.

To our knowledge, this is the first study monitoring therapy with vedolizumab by CEUS quantification; prior studies focused on patients mainly with $\mathrm{CD}$, and rarely with UC, upon treatment with anti-TNF agents. Using a linear transducer, CEUS at baseline and one, three, and 12 months after initiation of CD treatment $(n=14$, thereof six patients with treatment failure) showed a significant difference between responders and non-responders for the amplitude-based parameters (18). Socaciu et al. (19) performed CEUS in $\mathrm{CD}$ and UC patients before and after three months of treatment in correlation to endoscopic scores (a ROI in the abdominal muscle served as a reference). Quaia et al. (20) defined responders and non-responders 10-12 weeks after initiation of treatment by clinical activity staging and endoscopy in $50 \mathrm{CD}$ patients and performed ileal CEUS quantification at week 6. In 11/15 patients with UC, increasing histopathological inflammation score showed a trend toward higher PE values $(P=0.07)$ (21). Overall, these studies showed that amplitude-based parameters (such as PE or AUC) change during therapy, in particular a significant decrease was demonstrated in case of therapy response (proved by clinical scoring or endoscopy). Generally, the time-related variables as well as bowel wall thickness did not differ significantly between groups at any point. In $\mathrm{CD}$, considering a cut-off of $\leq-52.2 \%$ for change in $\mathrm{PE}$, a sensitivity of $77.8 \%$ and a specificity of $100 \%$ with an AUC of 0.893 were noted (20).

Most of these findings are in accordance with our results: a decrease of amplitude-derived parameters, in particular PE, WiR (statistically significant), and WoR in responders was observed. The changes in CEUS parameters during treatment with vedolizumab tended to be more pronounced in patients with $\mathrm{CD}$ than with UC. Wall thickness and time-derived parameters did not show any correlation.

The power of our findings is impaired by certain weaknesses that we share with other authors. In our study, therapeutic response was classified by slight changes in the clinical inflammation scores and not systematically to endoscopic findings that were not available in all patients (18). Thereby studies use different scoring systems for endoscopic or histopathological activity classification and time range of the endoscopic date is variable or not always described (19). Also, the definition of clinical improvement varies between the studies (22) and may be questioned in our cohort. Most patients had a long history of IBD and prior therapy with anti-TNF agents, sometimes with vedolizumab as the final therapeutic option, thus already slight improvements of disease activity were classified as response. The mechanisms of hypervascularization and blood flow are complex and may be variable in our study due to the relatively long course of disease. The patient number is quite small as vedolizumab was recently introduced, and we evaluated both UC and CD. Moreover, a relevant number of patients $(47 \%)$ had to be excluded, often due to problems with software-assisted analysis of video loops due to gas within the bowel lumen or thin bowel walls, beside interruption of therapy - in other studies exclusion rates have been reported in the range of $23-66 \%$ also due to technical problems or loss of follow-up $(19,20)$. In addition, there is no standardized algorithm of analysis. Some studies use a high-frequency linear transducer, whereas we used a convex probe due to its specific potential to detect the microbubbles. The lengths of the video loops may be different. We analyzed bowel wall vascularization directly with one ROI. Others propose a reference ROI in the mesenteric tissue or the iliac artery $(18,19)$. A recent study revealed differences in CEUS parameters between two different high-end ultrasound systems intra-individually in patients with CD (23). Moreover, a certain intra- and inter-observer bias cannot be excluded: the ROI during CEUS may be on a slightly different location than the evaluation of the b-mode parameters. Defining a ROI is very subjective concerning size and shape and may have affected the 
calculations. In addition, the definition of contrast arrival time depending on the observer's choice may affect time-dependent parameters. In the current state, software-based analysis of CEUS of the bowel wall seems not to be ready for use in daily clinical routine.

Limitations of our pilot study were the small number of analyzed patients; $47 \%$ patients from the screened cohort needed to be excluded. Patients suffered mostly from complex disease (long course of disease, vedolizumab as final therapeutic option). Therapeutic outcome was determined by clinical activity. An intra- and interobserver bias during high frequency b-mode ultrasound and CEUS analysis may have occurred. Due to these limitations, a direct comparison between study findings might be difficult. Nevertheless, our data are similar to observations found in the literature based on patients treated with anti-TNF agents.

In conclusion, bowel wall perfusion changes under treatment with vedolizumab. A decrease of semi-quantitative color Doppler flow and of amplitude-derived parameters by the sophisticated software-based analysis of CEUS is shown in treatment responders, whereas higher or relatively stable values were found in non-responders.

\section{Declaration of Conflicting Interests}

The author(s) declared the following potential conflicts of interest with respect to the research, authorship, and/or publication of this article: Goertz, Wildner and Strobel received fees for lecturing tutor in workshops about contrast-enhanced ultrasound (Bracco); Neurath is a consultant (Advisory Boards) for Takeda; Atreya received fees for lectures from Takeda.

\section{Funding}

The author(s) received the following financial support for the research, authorship, and/or publication of this article: Society of Gastroenterology in Bavaria (GFGB).

\section{References}

1. Schreiber S, Dignass AU, Hartmann H, et al. Current position on Vedolizumab for ulcerative colitis and Crohn's disease. Z Gastroenterol 2015;53:591-602.

2. Ripolles T, Paredes JM, Martinez-Perez MJ, et al. Ultrasonographic changes at 12 weeks of anti-TNF drugs predict 1-year sonographic response and clinical outcome in Crohn's disease: a multicenter study. Inflamm Bowel Dis 2016;22:2465-2473.

3. Bavil AS, Somi MH, Nemati M, et al. Ultrasonographic evaluation of bowel wall thickness and intramural blood flow in ulcerative colitis. ISRN Gastroenterol 2012;2012: 370495.

4. Limberg B. Diagnosis of chronic inflammatory bowel disease by ultrasonography. Z Gastroenterol 1999;37: 495-508.
5. Serra C, Menozzi G, Labate AM, et al. Ultrasound assessment of vascularization of the thickened terminal ileum wall in Crohn's disease patients using a lowmechanical index real-time scanning technique with a second generation ultrasound contrast agent. Eur J Radiol 2007;62:114-121.

6. Migaleddu V, Scanu AM, Quaia E, et al. Contrastenhanced ultrasonographic evaluation of inflammatory activity in Crohn's disease. Gastroenterology 2009;137: 43-52.

7. Tranquart F, Mercier L, Frinking $\mathrm{P}$, et al. Perfusion quantification in contrast-enhanced ultrasound (CEUS)-ready for research projects and routine clinical use. Ultraschall Med 2012;33(Suppl 1): S31-38.

8. Quaia E, Migaleddu V, Baratella E, et al. The diagnostic value of small bowel wall vascularity after sulfur hexafluoride-filled microbubble injection in patients with Crohn's disease. Correlation with the therapeutic effectiveness of specific anti-inflammatory treatment. Eur J Radiol 2009;69:438-444.

9. Piscaglia F, Bolondi L. The safety of Sonovue in abdominal applications: retrospective analysis of 23188 investigations. Ultrasound Med Biol 2006;32:1369-1375.

10. Harvey RF, Bradshaw JM. A simple index of Crohn'sdisease activity. Lancet 1980;1:514.

11. Schroeder KW, Tremaine WJ, Ilstrup DM. Coated oral 5-aminosalicylic acid therapy for mildly to moderately active ulcerative colitis. A randomized study. N Engl J Med 1987;317:1625-1629.

12. Quaia E MV, Baratella E, et al. The diagnostic value of small bowel vascularity after sulfur hexaflouride-filled microbubble injection in patients with Crohn's disease. Correlation with the therapeutic effectiveness of specific anti-inflammatory treatment. J Radiol 2009;2009: 438-444.

13. Parente F, Greco S, Molteni M, et al. Role of early ultrasound in detecting inflammatory intestinal disorders and identifying their anatomical location within the bowel. Aliment Pharmacol Ther 2003;18:1009-1016.

14. Heyne R, Rickes S, Bock P, et al. Non-invasive evaluation of activity in inflammatory bowel disease by power Doppler sonography. Z Gastroenterol 2002;40:171-175.

15. Girlich C, Schacherer D, Jung EM, et al. Comparison between a clinical activity index (Harvey-BradshawIndex), laboratory inflammation markers and quantitative assessment of bowel wall vascularization by contrast-enhanced ultrasound in Crohn's disease. Eur J Radiol 2012;81:1105-1109.

16. Romanini L, Passamonti $\mathrm{M}$, Navarria $\mathrm{M}$, et al. Quantitative analysis of contrast-enhanced ultrasonography of the bowel wall can predict disease activity in inflammatory bowel disease. Eur J Radiol 2014;83: 1317-1323.

17. Ripolles T, Rausell N, Paredes JM, et al. Effectiveness of contrast-enhanced ultrasound for characterisation of intestinal inflammation in Crohn's disease: a comparison with surgical histopathology analysis. J Crohns Colitis 2013;7:120-128.

18. Saevik F, Nylund K, Hausken T, et al. Bowel perfusion measured with dynamic contrast-enhanced ultrasound 
predicts treatment outcome in patients with Crohn's disease. Inflamm Bowel Dis 2014;20:2029-2037.

19. Socaciu M, Ciobanu L, Diaconu B, et al. Non-invasive assessment of inflammation and treatment response in patients with Crohn's disease and ulcerative colitis using contrast-enhanced ultrasonography quantification. J Gastrointestin Liver Dis 2015;24:457-465.

20. Quaia E, Sozzi M, Angileri R, et al. Time-intensity curves obtained after microbubble injection can be used to differentiate responders from nonresponders among patients with clinically active Crohn disease after 6 weeks of pharmacologic treatment. Radiology 2016;281: 606-616.

21. Girlich C, Schacherer D, Jung EM, et al. Comparison between quantitative assessment of bowel wall vascularization by contrast-enhanced ultrasound and results of histopathological scoring in ulcerative colitis. Int J Colorectal Dis 2012;27:193-198.

22. Quaia E, Cabibbo B, De Paoli L, et al. The value of timeintensity curves obtained after microbubble contrast agent injection to discriminate responders from nonresponders to anti-inflammatory medication among patients with Crohn's disease. Eur Radiol 2013;23: 1650-1659.

23. Zink F, Kratzer W, Schmidt S, et al. Comparison of two high-end ultrasound systems for contrast-enhanced ultrasound quantification of mural microvascularity in Crohn's disease. Ultraschall Med 2016;37:74-81. 